\title{
String/frets contacts in the electric bass sound: Simulations and experiments.
}

\author{
Clara Issanchou ${ }^{\mathrm{a}, *}$, Jean-Loïc Le Carrou $^{\mathrm{a}}$, Cyril Touzé $^{\mathrm{b}}$, Benoît Fabre ${ }^{\mathrm{a}}$, Olivier Doaré ${ }^{\mathrm{b}}$ \\ ${ }^{a}$ Sorbonne Universités, UPMC Univ Paris 06, CNRS, LAM/D'Alembert, 4 place Jussieu, 75252 Paris cedex 05, \\ France \\ ${ }^{b}$ IMSIA, ENSTA ParisTech-CNRS-EDF-CEA, Université Paris Saclay, Palaiseau, 91762 Cedex France
}

\begin{abstract}
For particular playing techniques such as "pop" or "slap" in the electric bass guitar, the string collides with frets, producing a percussive sound used in different music styles. The string/frets contacts introduce a non-linearity which is investigated both numerically and experimentally in this paper. A physical model, based on a modal description of the string, is implemented with an unconditionally stable scheme. Simulations including a string/structure coupling and the two polarisations of the string are confronted to controlled experiments, showing a good agreement for increasing amplitudes of initial conditions. A parametric study is then conducted numerically in order to highlight the influence of physical parameters on the transient behaviour and raises questions related to tuning and playing issues.
\end{abstract}

Keywords: Numerical methods, 3D string vibration, experimental study, unilateral contact, sound synthesis, electric bass guitar

\section{Introduction}

The solid-body electric bass has a recent history, opening up during the first half of the 20th century [1]. Originally designed to increase the sound level and to be played with better precision than the double bass, the solid-body electric bass was inspired by the solid-body electric guitar with four heavy strings tuned to the same notes as the double bass [1]. The sound of the instrument is a result of an electro-acoustic chain beginning with the string vibratory motion. This latter is then of prime importance and can be disrupted by its coupling with the structure of the instrument. The string vibration decay can vary depending on the finger position, due to the induced boundary condition, and dead spots can be produced at a fingering position. This phenomenon may be explained through a linear description of the coupling between the neck and the string [2, 3. However, possible nonlinear features are not investigated in these studies. In particular, among playing techniques adopted by musicians, some rely on a percussive aspect of the sound, implying contacts between vibrating strings and the neck. Two typical such playing modes are "pop", for which the string is plucked hardly enough to generate contact, and "slap", for which the string is hit with the thumb, also resulting in string/neck contacts [4]. The string/obstacle contact introduces nonlinearity, that has been widely studied numerically. The highly nonlinear behaviour of the string vibrating in presence of an obstacle makes the problem stiff and implies numerical difficulties, in particular regarding stability. Among existing numerical methods, some models use waveguides [5, 6, 7, which reproduce effects through signal processing, or energy-based methods [8, 9, 10, 11, ensuring a good stability to employed schemes. Some models rely on a modal description of the string [12, 13, 10, 11, which possibly enables a fine description of the string linear characteristics such as damping. Only a few studies present experimental signals with an isolated string or a complete instrument in order to give a comparison point for their simulations [12, 14, 11, 15, the latter being applied to slap on electric basses. In [16, a listening test is performed to evaluate the synthesis algorithm.

The present paper aims at presenting a numerical tool to simulate musical strings vibrating against a unilateral distributed obstacle, and confronting it to experiments in detail. The method is applied

\footnotetext{
${ }^{*}$ Corresponding author

Email address: issanchou@lam.jussieu.fr (Clara Issanchou )
} 
to the pop attack on electric basses, for which the string is plucked with a sufficiently large amplitude so that contact occurs and gives the sound a percussive timbre during the attack transient. The objective of such a numerical tool is to move forward the comprehension of the string behaviour when colliding with a fretboard, through the study of some key parameters. The employed numerical model is presented in Section 2, A controlled experimental protocol is then proposed in Section 3 , Numerical and experimental signals are confronted in Section 4 and a numerical parametric study is led in order to highlight the influence of some parameters on the resulting sound, some of which may be related to playing and instrument making issues.

\section{Model}

\subsection{Model of a string vibrating against an obstacle}

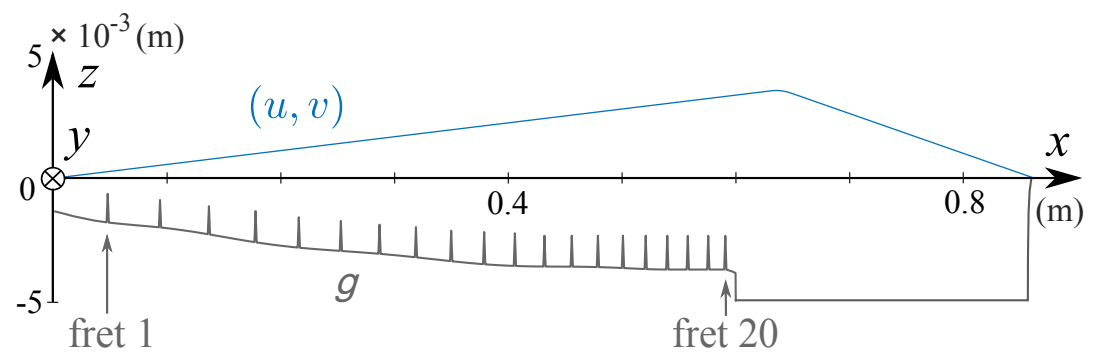

Figure 1: A string of length $L$ vibrating against a bass guitar fretboard represented by the function $g$.

A stiff string of length $L$, mass per unit length $\mu$, tension $T$, Young's modulus $E$ and moment of inertia $I$ is considered. The string (see Fig. 1) vibrates in the presence of an obstacle having a profile $g(x)$ which is constant along $(O y)$ and under the string at rest. The transverse displacements $u(x, t)$ and $v(x, t)$ of the string along $(O z)$ and $(O y)$ respectively are described by Eq. (1), in which the subscript $t$ (respectively $x$ ) refers to a partial derivative with respect to time (respectively space):

$$
\begin{aligned}
& \mu u_{t t}-T u_{x x}+E I u_{x x x x}=f \\
& \mu v_{t t}-T v_{x x}+E I v_{x x x x}=f_{f},
\end{aligned}
$$

where the right-hand sides $f$ (contact force per unit length) and $f_{f}$ (friction force per unit length) are fully described later.

Simply supported boundary conditions at the string endpoints are employed, this corresponds to a common assumption for musical strings with a weak stiffness, see e.g. [12, 17. For the sake of conciseness, the next equations are only detailed for the vertical displacement $u$, but of course also apply to the horizontal displacement $v$. Boundary conditions read, $\forall t \in \mathbb{R}^{+}$:

$$
u(0, t)=u(L, t)=u_{x x}(0, t)=u_{x x}(L, t)=0 .
$$

The displacement is then spatially discretised by using $N_{m}$ eigenmodes:

$$
u(x, t)=\sum_{j=1}^{N_{m}} q_{j}(t) \phi_{j}(x),
$$

with $\phi_{j}(x)=\sqrt{\frac{2}{L}} \sin \left(\frac{j \pi x}{L}\right)$ and $q_{j}$ the $j$ th modal amplitude.

Inserting this expression in Eq. (1a), using standard Galerkin projection technique and adding losses, one finally obtains a system of oscillators for the unknown $\mathbf{q}=\left[q_{1}, q_{2}, \ldots q_{N_{m}}\right]^{T}$ gathering the modal amplitudes as:

$$
\mu\left(\ddot{\mathbf{q}}+\mathbf{\Omega}^{2} \mathbf{q}+2 \Upsilon \dot{\mathbf{q}}\right)=\mathbf{F}
$$

where $\Omega$ and $\Upsilon$ are diagonal matrices with coefficients $\Omega_{j j}=\omega_{j}=2 \pi \nu_{j}, \nu_{j}$ being the $j$ th eigenfrequency, and $\Upsilon_{j j}=\sigma_{j}$, which corresponds to the $j$ th damping coefficient.

A penalty approach is selected to express the contact force per unit length, following [9, 11]:

$$
f(\eta(x, t))=K[\eta(x, t)]_{+}^{\alpha},
$$


where $\eta(x, t)=g(x)-u(x, t)$ represents the penetration of the string into the barrier, and $[\eta]_{+}=$ $\frac{1}{2}(\eta+|\eta|)$. The regularised contact force thus depends on two parameters $K$ and $\alpha$, and derives from a potential $\psi$ :

$$
f=\frac{d \psi}{d \eta}, \quad \text { where } \quad \psi(\eta)=\frac{K}{\alpha+1}[\eta]_{+}^{\alpha+1} .
$$

The friction force per unit length $f_{f}$, acting on the polarisation along $(O y)$, is selected as a regularised empirical Tresca friction law [18, 19], and reads:

$$
f_{f}\left(u, v_{t}\right)=A\left\{\begin{array}{l}
1 \quad \text { if } v_{t}<-s \text { and } u<g \\
-v_{t} / s \text { if }\left|v_{t}\right| \leq s \text { and } u<g \\
-1 \quad \text { if } v_{t}>s \text { and } u<g \\
0 \quad \text { if } u \geq g,
\end{array}\right.
$$

where $v_{t}$ is the transverse velocity of the string along $(O y)$, and $A\left(\mathrm{~N} . \mathrm{m}^{-1}\right), s>0\left(\mathrm{~m} . \mathrm{s}^{-1}\right)$ are two ad hoc parameters. A number of studies in the literature use a regularised friction force [20, 21. Note that such a friction force only allows one equilibrium position, this may lead to incorrect behaviours in some configurations 22 .

In order to take into account the vibrations of the neck, the mobility at the nut is then added to complete the model. In the case of solid body electric guitars and basses, it has been shown that the bridge mobility is negligible as compared to that at the nut [3, 2. Moreover, as detailed in Section 2.2, the coupling is weak so that taking into account the nut mobility only alters linear characteristics.

\subsection{Linear characteristics models}

The influence of the dispersion due to the stiffness of the string, though small, needs to be taken into account. Also, under the previously exposed assumption of weak coupling at the nut and as done in [3] for electric guitars, the eigenfrequencies are modeled following the relationship, for each mode $j$ :

$$
\nu_{j}=j \frac{c}{2 L}\left(1+\frac{B j^{2}}{2}+\frac{\mu c}{j \pi} \operatorname{Im}\left(Y_{n u t}\left(\omega_{0, j}\right)\right)\right),
$$

where $c=\sqrt{\frac{T}{\mu}}$ is the wave velocity of the ideal string, $B=\frac{\pi^{2} E I}{T L^{2}}$ is the inharmonicity coefficient and $Y_{n u t}$ is the mobility at the nut, evaluated at $\omega_{0, j}=j \frac{\pi c}{L}$. In the present study $B$ is deduced from measurements (see Section 3.2).

The modal loss factor is evaluated thanks to the model exposed in [12, 3]. For mode $j$, the quality factor $Q_{j}$ is used to express the modal damping factor $\sigma_{j}$ via $Q_{j}=\pi \nu_{j} / \sigma_{j}$, where $Q_{j}$ is modeled as:

$$
Q_{j}^{-1}=Q_{j, \text { air }}^{-1}+Q_{j, \mathrm{ve}}^{-1}+Q_{\mathrm{te}}^{-1}+\frac{\mu c^{2}}{\pi L \nu_{j}} \operatorname{Re}\left(Y_{n u t}\left(\omega_{j}\right)\right)
$$

In this model, the subscripts air, ve and te respectively refer to losses due to air friction, viscoelastic and thermoelastic effects.

Contribution of friction with air writes:

$$
Q_{j, \text { air }}^{-1}=\frac{j c}{2 L \nu_{j}} \frac{R}{2 \pi \mu \nu_{j}}
$$

where $R=2 \pi \eta_{\text {air }}+2 \pi d_{e q} \sqrt{\pi \eta_{\text {air }} \rho_{\text {air }} \nu_{j}}$, with $\eta_{\text {air }}$ and $\rho_{\text {air }}$ the dynamic viscosity coefficient and the air density respectively. Usual values (for standard temperature and pressure conditions) are selected here as: $\eta_{\text {air }}=1.8 \times 10^{-5} \mathrm{~kg} \cdot \mathrm{m}^{-1} . \mathrm{s}^{-1}$ and $\rho_{\text {air }}=1.2 \mathrm{~kg} \cdot \mathrm{m}^{-3}$. Viscoelastic effects are supposed to be concentrated in the string core [12], so that their contribution to global losses is given by:

$$
Q_{j, \mathrm{ve}}^{-1}=\frac{4 \pi^{2} \mu E_{\text {core }} I_{\text {core }} \delta_{\mathrm{ve}}}{T^{2}} \frac{\nu_{0, j}^{3}}{\nu_{j}},
$$

where $E_{\text {core }}$ is the Young's modulus of the core, $I_{\text {core }}=\pi r_{\text {core }}^{4} / 4$ is the moment of inertia of the core, with $r_{\text {core }}$ the core radius, and $\nu_{0, j}=\frac{j c}{2 L}\left[12\right.$. Finally, the viscoelastic loss angle $\delta_{\mathrm{ve}}$ and the constant value $Q_{\mathrm{te}}^{-1}$ may be adjusted according to measurements. 


\subsection{Numerical model}

The numerical model used in this contribution has been first introduced in [11] where the interested reader can find a detailed description. Here only the salient features are briefly exposed.

The numerical scheme combines an exact solution for the linear part (when no contact occurs) with an energy-conserving expression for the regularised contact force. More specifically, the equation of motion is first expressed in terms of the vector $\mathbf{u}=\left[u_{1}, u_{2}, \ldots u_{N-1}\right]^{T}$ of the string displacement on interior points of an equally distributed spatial grid. Assuming a number of modes equal to the number of interior points of the spatial grid, i.e. $N_{m}=N-1$, a simple relationship exists between $\mathbf{u}$ and the vector of modal coefficients $\mathbf{q}=\left[q_{1}, q_{2}, \ldots q_{N-1}\right]^{T}: \mathbf{u}=\mathbf{S q}$, where $\mathbf{S}$ has entries $S_{i j}=\phi_{j}\left(x_{i}\right)$, $\forall(i, j) \in\{1, \ldots, N-1\}^{2}$. The discrete equation on $\mathbf{q}$ can therefore easily be rewritten for $\mathbf{u}$. Then the contact force is discretised according to the expression suggested in [9].

The scheme on $\mathbf{u}$ finally writes:

$$
\frac{\mu}{\Delta t^{2}}\left(\mathbf{u}^{n+1}-\mathbf{D} \mathbf{u}^{n}+\tilde{\mathbf{D}} \mathbf{u}^{n-1}\right)=\mathbf{f}^{n}
$$

with $\mathbf{f}^{n}=\frac{\psi\left(\eta^{n+1}\right)-\psi\left(\eta^{n-1}\right)}{\eta^{n+1}-\eta^{n-1}}, \mathbf{D}=\mathbf{S C S}^{-1}$ and $\tilde{\mathbf{D}}=\mathbf{S} \tilde{\mathbf{C}} \mathbf{S}^{-1} . \mathbf{C}$ and $\tilde{\mathbf{C}}$ are diagonal matrices with entries:

$$
\begin{aligned}
C_{i i} & =\mathrm{e}^{-\sigma_{i} \Delta t}\left(\mathrm{e}^{\sqrt{\sigma_{i}^{2}-\omega_{i}^{2}} \Delta t}+\mathrm{e}^{-\sqrt{\sigma_{i}^{2}-\omega_{i}^{2}} \Delta t}\right), \\
\tilde{C}_{i i} & =\mathrm{e}^{-2 \sigma_{i} \Delta t},
\end{aligned}
$$

where $\Delta t$ is the time step, with corresponding sampling frequency $F_{s}=1 / \Delta t$.

The discrete equation for the displacement $\mathbf{v}=\left[v_{1}, v_{2}, \ldots v_{N-1}\right]^{T}$ along $(O y)$ is obtained in a similar way:

$$
\frac{\mu}{\Delta t^{2}}\left(\mathbf{v}^{n+1}-\mathbf{D} \mathbf{v}^{n}+\tilde{\mathbf{D}} \mathbf{v}^{n-1}\right)=f_{f}\left(\mathbf{u}^{n}, \delta_{t} \cdot \mathbf{v}^{n}\right)
$$

where $\delta_{t} . \mathbf{v}^{n}=\frac{\mathbf{v}^{n+1}-\mathbf{v}^{n-1}}{2 \Delta t}$.

This scheme is conservative if there is no damping, dissipative otherwise, and unconditionally stable [11. It should also be noted that the scheme is implicit, and that a Newton-Raphson algorithm is used at each time step to solve the nonlinear equation for updating the unknowns. The computation costs associated to each operation in the time-stepping algorithm have been fully discussed in [23, showing in particular that the most time-consuming part was the computation of products between full matrices and vectors instead of the Newton loop.

The proposed scheme thus combines a modal approach together with a treatment of the contact force in the physical domain, in order to process the different terms of the dynamical equations in the most convenient space (modal or physical), as done for instance in [10, 24]. This method also allows one to use an exact scheme for the instants without contact, and an easy to handle conservative numerical expression of the contact force. The drawback of the present scheme is the use of an equal number of modes and grid points, which may lead to consider a large number of modes in the truncation. Decoupling these two numbers is nonetheless possible, as done for example in [10].

\section{Experimental characterisation}

\begin{tabular}{|c|c|c|c|c|}
\hline$L(\mathrm{~m})$ & $d(\mathrm{~mm})$ & $d_{\text {core }}(\mathrm{mm})$ & $T(\mathrm{~N})$ & $\mu\left(\mathrm{kg} . \mathrm{m}^{-1}\right)$ \\
\hline 0.863 & 1.14 & 0.43 & 191.6 & $6.69 \times 10^{-3}$ \\
\hline
\end{tabular}

Table 1: String properties

The G-string of an electric bass with fundamental frequency $98 \mathrm{~Hz}$ is considered. Its properties are specified in Table 1, $T$ being deduced from the other parameters. It is installed on a Fender Jazz Bass, itself put on elastics in order to simulate free boundary conditions. Only the open string is investigated here. 


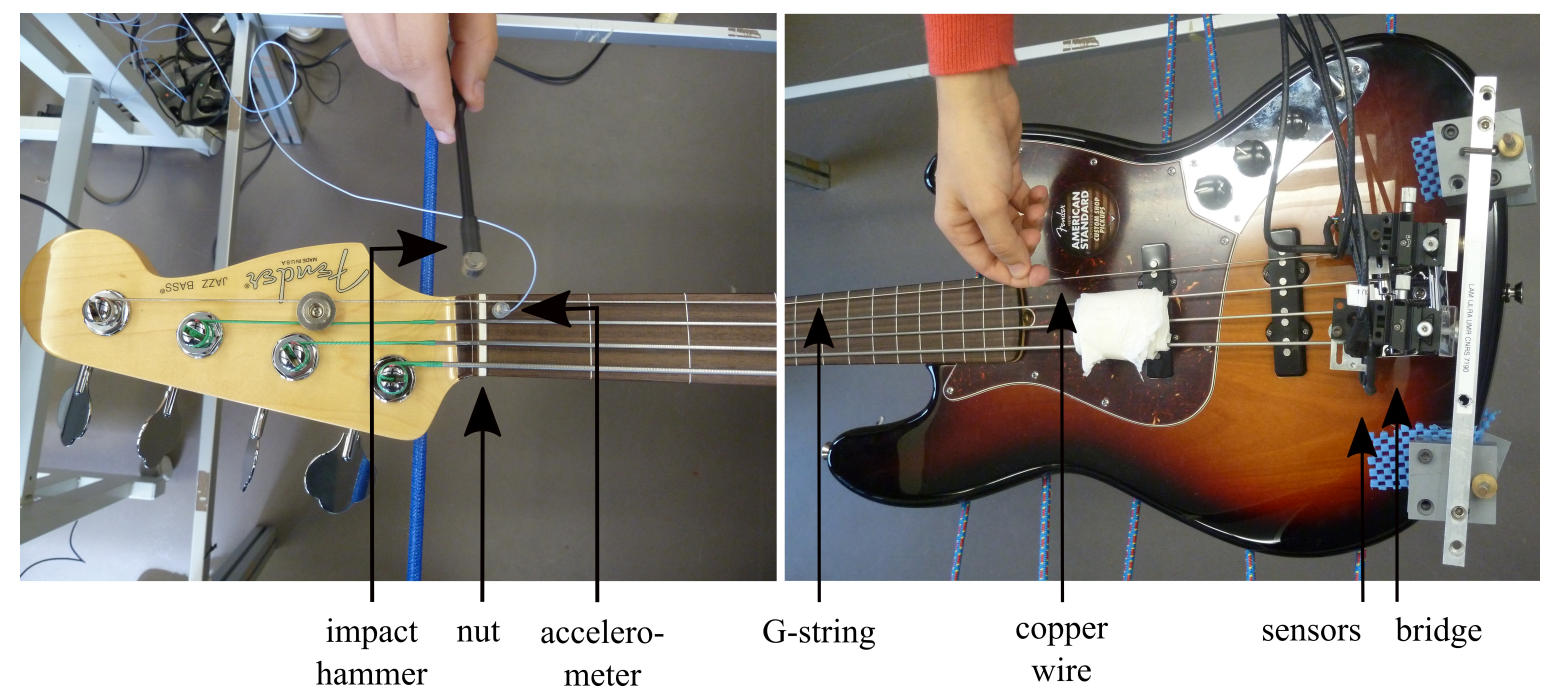

Figure 2: Experimental set up.

\subsection{Experimental set up}

The mobility at the nut is measured by applying an impulse force and recording the associated acceleration. To this end, a Brüel \& Kjær 8203 impact hammer together with a PCB Piezotronics 352B10 accelerometer are employed (see Fig. 2).

The string displacement is obtained thanks to optical sensors calibrated according to the procedure described in [25. Uncertainties are obtained using the theoretical expression given in 25. Both displacements along $(O z)$ and $(O y)$ are recorded simultaneously with sensors located respectively at 9 and $18 \mathrm{~mm}$ from the bridge, and the neck profile is measured using a ruler. The initial condition is provided by pulling the string with a copper wire until it breaks, at a selected location $(64 \mathrm{~cm}$ from the nut for free vibration measurements, see Fig. 2 and $2 \mathrm{~cm}$ from the nut in the next section for identification of linear characteristics), as done e.g. in [3, 11. Different diameters of copper wire $(0.05,0.1$ and $0.15 \mathrm{~mm})$ allow one to get initial conditions with increasing amplitudes. Signals are obtained with a sampling rate of $51200 \mathrm{~Hz}$. In order to avoid sympathetic vibrations [26], other strings are damped. The fretboard profile, presented in Fig. 1 is typical from a standard neck adjustment according to musicians (private communications).

\subsection{Identification of linear characteristics}

In order to identify the linear characteristics of the string coupled to the instrument, it is plucked near the nut with a $0.05 \mathrm{~mm}$ diameter wire, so that a large number of modes are excited and no contact arises. Then a high resolution signal processing method is employed which implies the ESPRIT algorithm [27, according to the procedure described in [28, 3, 11].

Table 2: Models parameters

\begin{tabular}{|c|c|c|c|c|c|}
\hline$B$ & $\delta_{\text {ve }}$ & $Q_{\mathrm{te}}^{-1}$ & $\alpha$ & $K$ & $N$ \\
\hline $3.5 \times 10^{-5}$ & 0.01 & $6 \times 10^{-6}$ & 1.5 & $10^{13}$ & 863 \\
\hline
\end{tabular}

The results are reported in Fig. 3. Uncertainties are computed over about ten measurements. The models presented in Section 2.2 are also reported, where the quality factor is shown with and without taking the mobility into account in Fig. 3. b). As it can be observed, the mobility substantially affects the quality factor of the string in the low frequency range (from about 100 to $2000 \mathrm{~Hz}$ ). The parameters of the eigenfrequencies and damping models are specified in Table 2 , they are selected in order to obtain the best fit with measurements, in particular for highest frequencies, from the 20th to the 34th mode. The mobility at the nut is included in the models up to about $10000 \mathrm{~Hz}$; note that its contribution to eigenfrequencies is negligible, and its contribution to damping decreases with frequency and becomes negligible beyond the 30 th mode. 


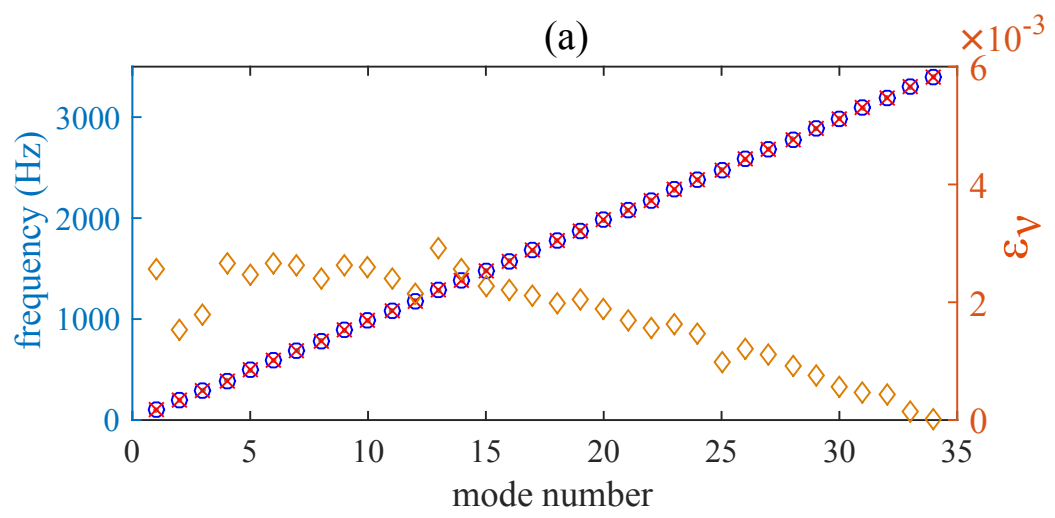

(b)

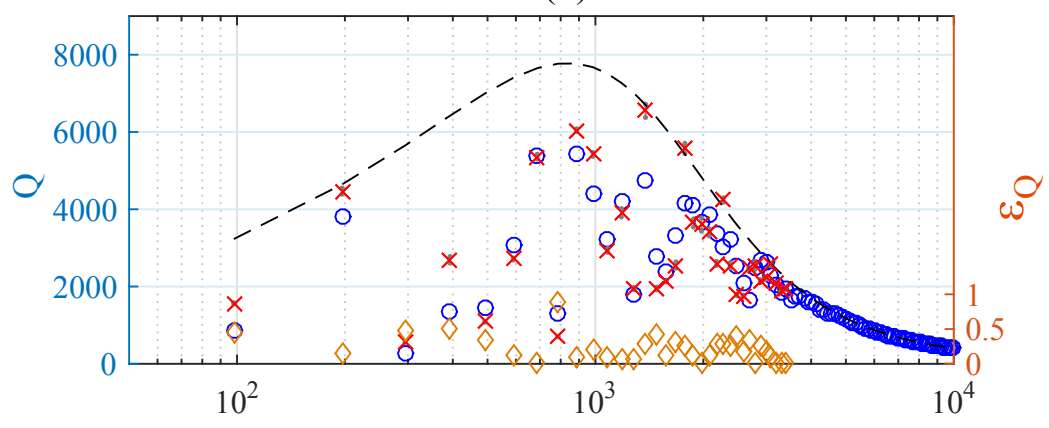

frequency $(\mathrm{Hz})$

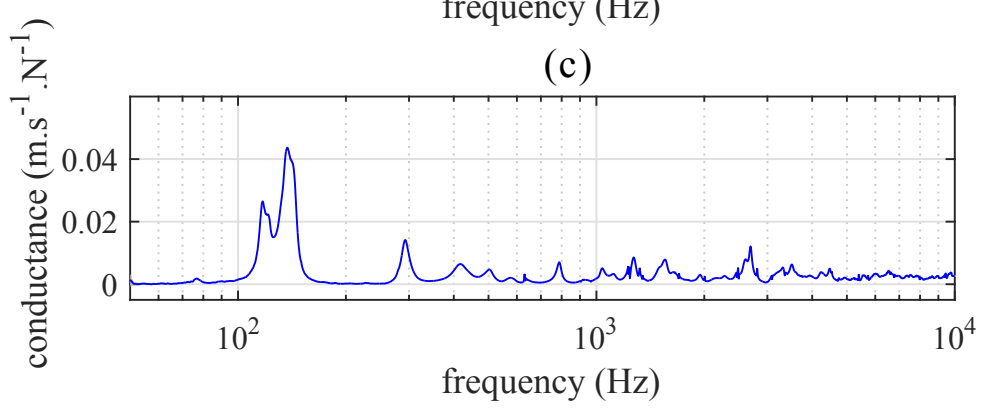

Figure 3: (a) Experimental (red crosses) and theoretical (blue circles) eigenfrequencies, $\nu_{m}$ and $\nu_{t h}$ respectively, expanded uncertainty at $95 \%$ (gray lines) and relative error (orange diamonds) $\epsilon_{\nu}=\frac{\left|\nu_{t h}-\nu_{m}\right|}{\nu_{m}}$. (b) Experimental (red crosses) and theoretical (blue circles) quality factors, $Q_{m}$ and $Q_{t h}$ respectively, theoretical quality factors without mobility (dark dashed line), expanded uncertainty at $95 \%$ (gray lines) and relative error (orange diamonds) $\epsilon_{Q}=\frac{\left|Q_{t h}-Q_{m}\right|}{Q_{m}}$. (c) Conductance at the nut (blue line) and expanded uncertainties at $95 \%$ (gray). Uncertainties are barely visible at this scale as they are very small as compared to the measured values.

In order to obtain the most precise input parameters in the numerical model, measured values of the linear characteristics are directly included up to the 34 th mode, i.e. around $3400 \mathrm{~Hz}$. Beyond this value, the models exposed in Section 2.2 are employed.

\section{Results}

In this section, numerical results and experimental data are confronted without and with contact. Numerical simulations are conducted with parameters specified in Table 2. The sampling frequency $F_{s}$ depends on the case tested and the number of contacts involved. A convergence criterion is selected as a relative error of the signal evaluated at the measurement point smaller than 0.1 , on a simulation duration that encompasses all contact times at least. This stringent criterion is necessary and leads to use large values of the sampling frequency in order to determine properly the high frequency content of the signals generated by impacts. 


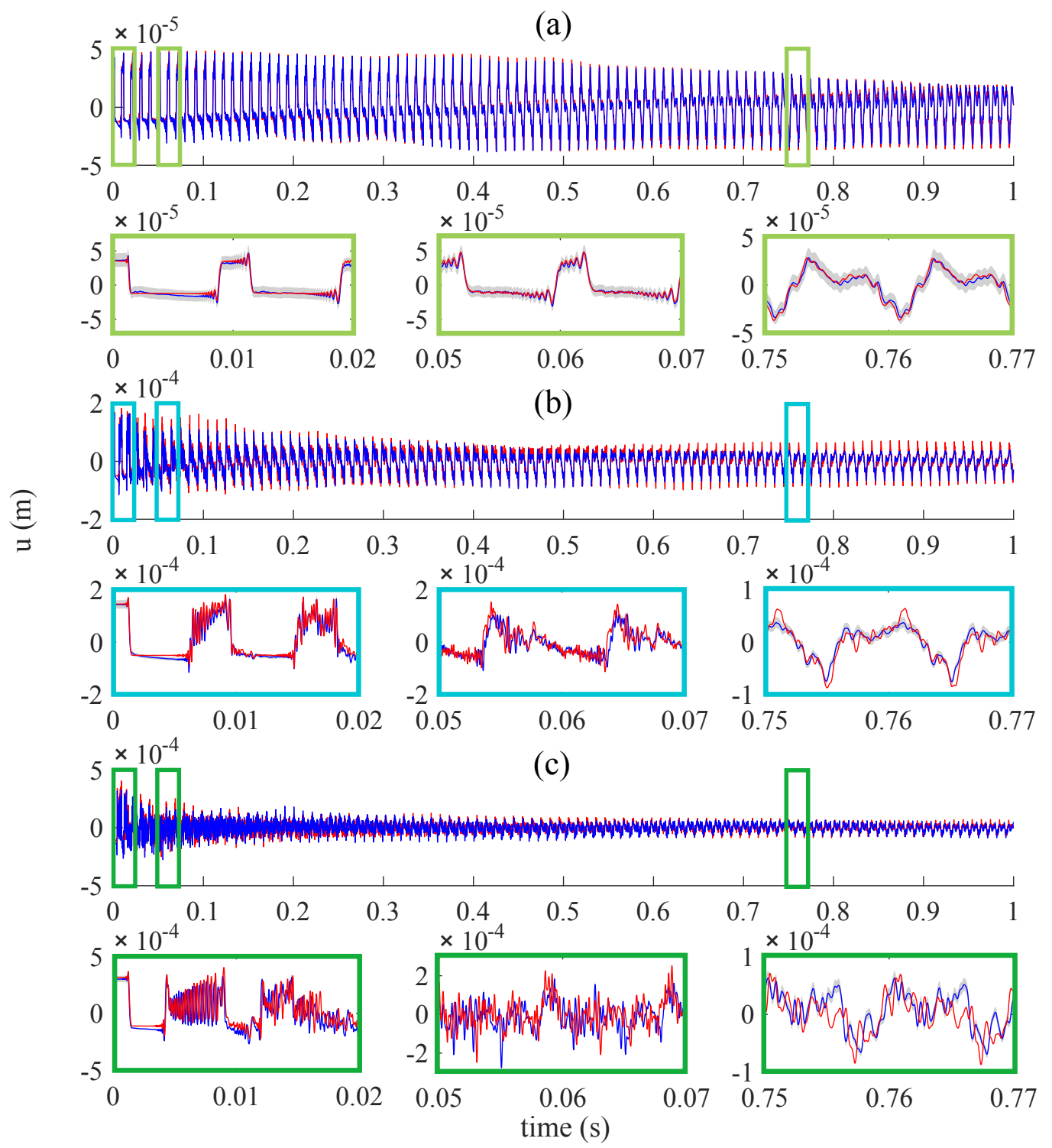

Figure 4: Displacement of the string: $1 \mathrm{~s}$ length signals and zooms. Comparison between measurements (blue lines) and numerical simulations (red lines), the observed variable is the $(O z)$ displacement at $9 \mathrm{~mm}$ from the edge $x=L$. Expanded uncertainty at $95 \%$ (gray). (a) Case A: without contact, $u_{0, \max }=0.87 \mathrm{~mm}, F_{s}=51200 \mathrm{~Hz}$. (b) Case B: with contact, $u_{0, \max }=3.6 \mathrm{~mm}, F_{s}=8 \mathrm{MHz}$. (c) Case C: with contact, $u_{0, \max }=7.8 \mathrm{~mm}, F_{s}=16 \mathrm{MHz}$. 
For comparative listening tests complementing the analysis shown in the paper, all the sounds corresponding to either experiments or simulations can be heard from the webpage

http://www. lam.jussieu.fr/Membres/Issanchou/sounds_bass17. They correspond to the displacement of the string at the position of sensors, resampled at $44.1 \mathrm{kHz}$ (WAV format).

\subsection{Comparison of numerical simulations and measurements}

In this section, a comparison is drawn between experimental and numerical signals. The initial shape is a rounded triangle with an apex at $64 \mathrm{~cm}$ from the nut. The string is excited along the $(O z)$ polarisation only, by properly pulling it vertically with the copper wire. The measured displacement along $(O y)$ is found to be negligible, and is thus discarded in the simulation. Section 4.1.3 is devoted to initial conditions implying both transverse polarisations.

Three cases are considered. The first one concerns a sufficiently small maximal initial displacement $(0.87 \mathrm{~mm})$ so that no contact occurs. This case, labeled $\mathrm{A}$, is used to validate the identification procedure. Two others cases, labeled B and $\mathrm{C}$, imply contacts between the string and a large number of frets, depending on the maximal initial displacement, given as $3.6 \mathrm{~mm}$ and $7.8 \mathrm{~mm}$ respectively. These values have been selected as lying within the range of typical pop playing amplitudes.

Fig. 4 shows the temporal signals for the three cases considered. When no contact occurs, numerical and experimental signals show an almost perfect matching of global shapes as well as detailed waveforms, resulting in extremely similar frequency distributions and sounds. This strong agreement highlights the quality of the identification procedure and the versatility of the numerical method. A more thorough discussion is now dedicated to cases $\mathrm{B}$ and $\mathrm{C}$.

\subsubsection{Case B: with contact, $u_{0, \max }=3.6 \mathrm{~mm}$}

Case B corresponds to a maximal initial displacement of $3.6 \mathrm{~mm}$ and gives rise to a number of contacts during the vibration such that nonlinear effects are now involved. The global comparison shown in Fig. 4(b) is very satisfactory. One can notice that the period at the very beginning of the signal seems to be shorter than in case A, which would mean that the fundamental frequency is higher than in the rest of the signal. A time-frequency analysis is shown in Fig. 5(a-b) with spectrograms, revealing interesting effects and noteworthy similarities. The most salient feature corresponds to the complex energy transfers at the beginning of the signals, as long as contacts occur. This phenomenon lasts about $0.09 \mathrm{~s}$ during which a large amount of energy is transmitted to higher modes. It is well recovered by the numerical simulation even though it seems to last slightly longer in the experimental case. Besides, some reinforced spectral zones can be clearly identified, for instance around $4500 \mathrm{~Hz}$, which are also well reproduced numerically.

A more quantitative viewpoint on the energy transfer can be given by defining a characteristic frequency $\nu_{c}$ as [29]:

$$
\nu_{c}=\frac{\int_{\nu=0}^{F_{s} / 2} a(\nu)^{2} \nu d \nu}{\int_{\nu=0}^{F_{s} / 2} a(\nu)^{2} d \nu},
$$

where $a(\nu)$ is the Fourier amplitude evaluated at frequency $\nu$, for the signal resampled at $F_{s}=$ $51200 \mathrm{~Hz}$.

Fig. 6 shows the behaviour of $\nu_{c}$ in cases A, B and C. In case A, $\nu_{c}$ slightly decreases in time because of the larger damping coefficients of high frequency modes. In cases $\mathrm{B}$ and $\mathrm{C}$, the spectral enrichment occurring in the first instants is clearly visible, and more pronounced in case $\mathrm{C}$ for which the initial amplitude is larger. One can observe similar evolutions of $\nu_{c}$ between the experiment and the simulation. Relative errors between the experimental and numerical signals having characteristic frequencies $\nu_{c, m}$ and $\nu_{c, n}$ respectively, given by $\frac{\nu_{c, n}-\nu_{c, m}}{\nu_{c, m}}$, are below 0.2 over the full signals duration in case B.

A second interesting feature, revealed by the spectrograms and confirming a previous observation made on temporal signals, is a substantial frequency glide in the low-frequency range (mostly below $500 \mathrm{~Hz}$, see the zooms in Fig. 5(a-b)), which is faithfully reproduced numerically. This phenomenon, due to the presence of an obstacle, has already been observed in the case of a curved obstacle at one string extremity in [30]. In the present case, it can be closely related to the occurrence of contacts in space and time. To do so, a contactogram, which shows the contact times on frets for the numerical signal, is presented in Fig.7 7.a). More specifically, the contact times and their locations on the fretboard 
(a)
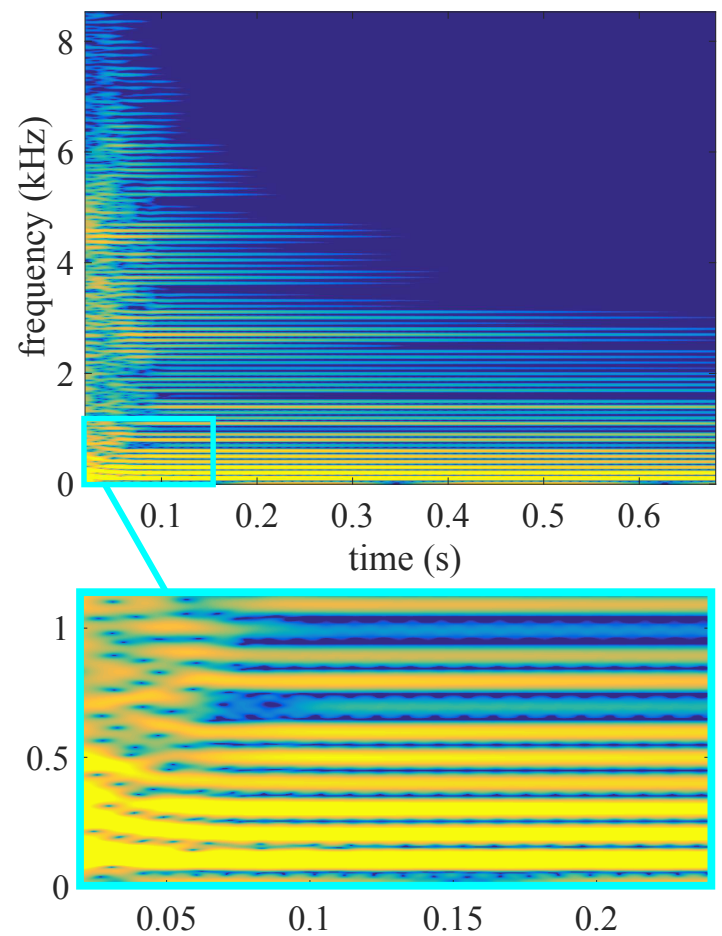

(c)

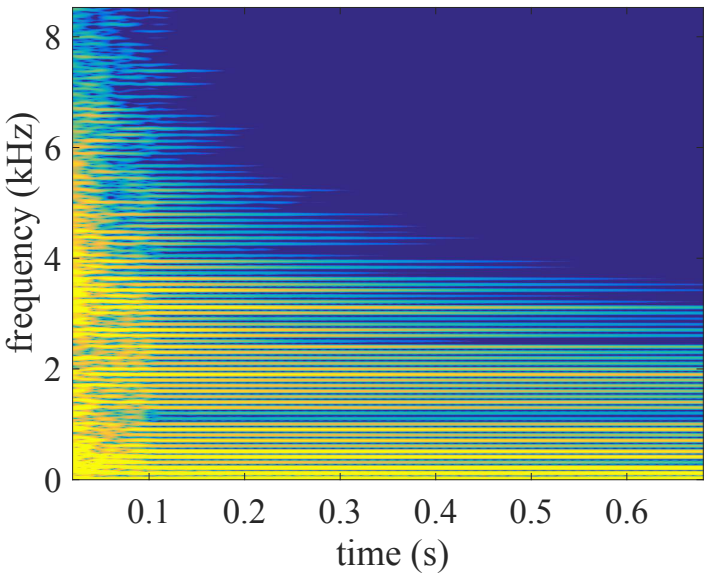

(b)

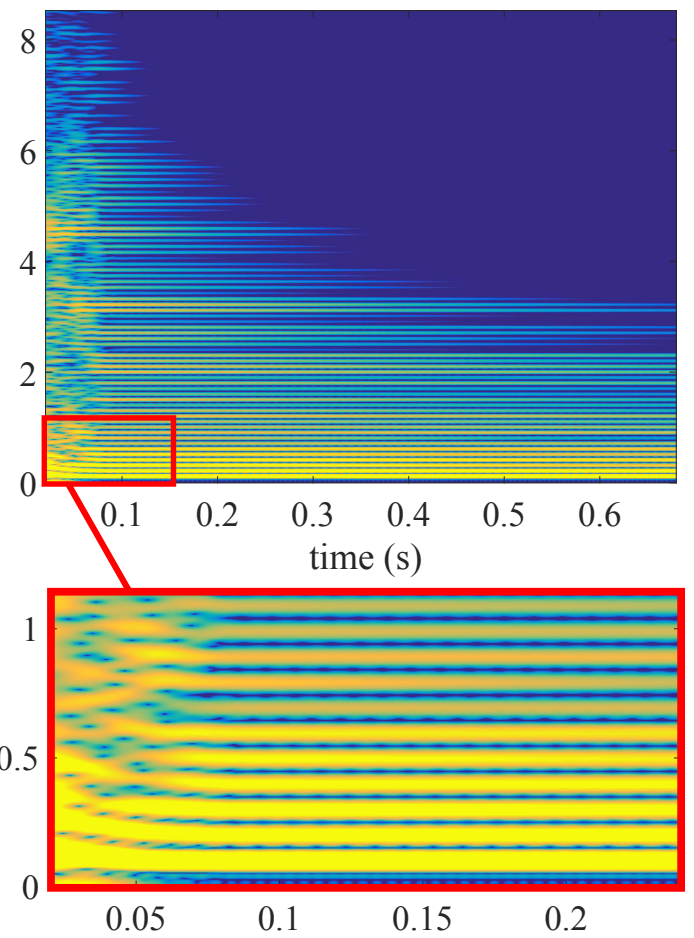

(d)

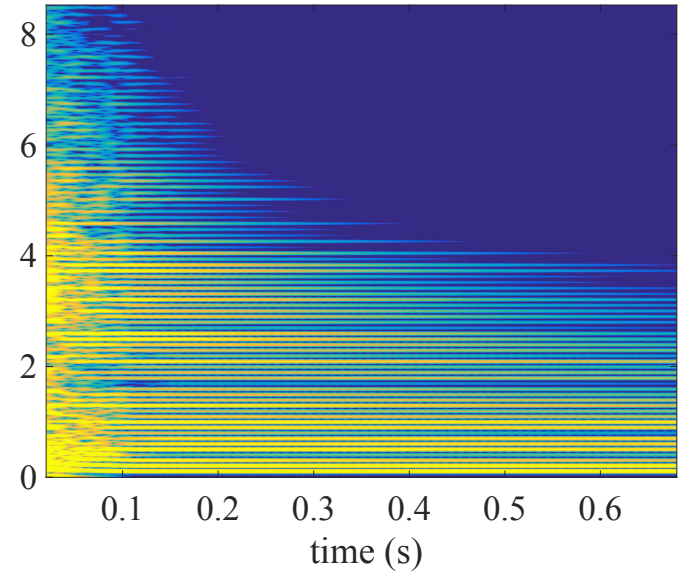

Figure 5: Spectrograms of the displacement in the case of string/frets contacts, drawn in dB using a $70 \mathrm{~dB}$ dynamic. (a) Experimental, with zoom, $u_{0, \max }=3.6 \mathrm{~mm}(\mathrm{~b})$ numerical, with zoom, $u_{0, \max }=3.6 \mathrm{~mm}, F_{s}=8 \mathrm{MHz}(\mathrm{c})$ experimental, $u_{0, \max }=7.8 \mathrm{~mm}(\mathrm{~d})$ numerical, $u_{0, \max }=7.8 \mathrm{~mm}, F_{s}=16 \mathrm{MHz}$. 


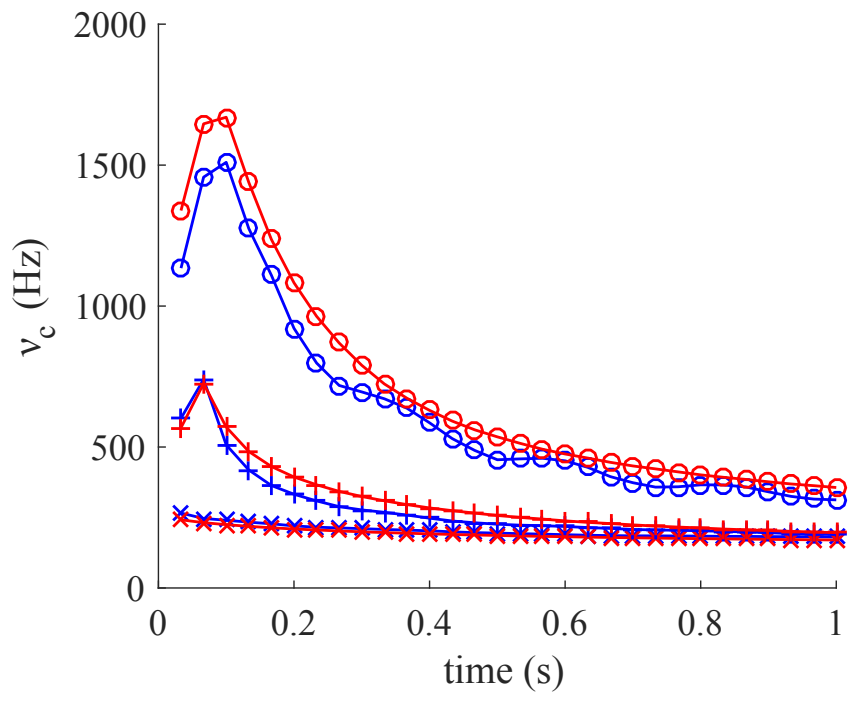

Figure 6: Characteristic frequencies of experimental (blue) and numerical (red) signals for increasing maximal initial displacements. Case A (lines with crosses), B (lines with plus signs) and C (lines with circles).

are detected from the numerical simulation and then reported as a function of the frets colliding with the string. The first set of collisions occurs around $4 \times 10^{-3} \mathrm{~s}$, and the string hits 12 frets (see Fig. 1). As shown in the zoom of the first contact time (insert of Fig. 7 (a)), the string first collides with the twelfth fret. For a better understanding, this first set of collisions may be observed together with Fig. 8, which shows the string shape at times included in this period. During the second set of collisions, the string touches frets 2 to 11 . Globally, less and less frets are hit. A peculiar feature in this simulation is that the first fret is hit at the first collision, and a second time long after, around $0.05 \mathrm{~s}$.

Superimposed to the contactogram in Fig. 7(a) is also shown the relative fundamental frequency variation during the attack transient, computed as $\frac{\nu(t)-\nu_{r}}{\nu_{r}}$, where $\nu_{r}$ is the first identified eigenfrequency $(98 \mathrm{~Hz})$, and $\nu(t)$ is the instantaneous fundamental frequency of the signal at time $t$, which has been obtained with the YIN algorithm [31, for both numerical and experimental signals. It allows one to obtain a more quantitative viewpoint on the fundamental frequency decrease already observed in spectrograms. More specifically, a staircase-like behaviour is found, where a decrease of the fundamental frequency arises each time the neck is hit. The frequency can be directly related to the number of touched frets: the smaller the number of hit frets is, the lower the frequency is. Once again, the agreement between the simulation and the experiment is very close when analysing the fundamental frequency variations.

Despite a global agreement which is very satisfactory, showing that the model has been able to reproduce faithfully all the main features of the experiment, some small differences still persist. A number of factors may explain these observations. In the model, the obstacle is assumed to be rigid while it has a mobility, not only at the nut (this is included in the model), but also on the full length of the neck. This may affect the string motion when contact occurs. Besides, there are uncertainties on each measurement step. Apart from those on eigenfrequencies, damping parameters and the plucking position, which appear to be small and controlled as revealed by analysing case A, the main measurement uncertainty concerns the neck profile and more specifically the location and height of each fret. In numerical simulations, it has been found that refining the space step did not significantly improve the results. However, it shows undoubtedly that the obtained sound is very sensitive to the height of the frets. This is illustrated through numerical examples in Section 4.2 .

\subsubsection{Case $C$ : with contact, $u_{0, \max }=7.8 \mathrm{~mm}$}

The case of a larger initial amplitude, such that $u_{0, \max }=7.8 \mathrm{~mm}$ (case $\mathrm{C}$ ), is finally investigated. The number of hit frets is larger than in case B, resulting in a more complex temporal signal displaying a considerable high-frequency content, as shown in Fig. 4(c). The agreement of experimental and numerical results is still very satisfactory, though differences are now a bit more pronounced than in case B. Spectrograms in Fig. 5. (c-d) show that the energy transfer to high frequencies is more substantial 
(a)

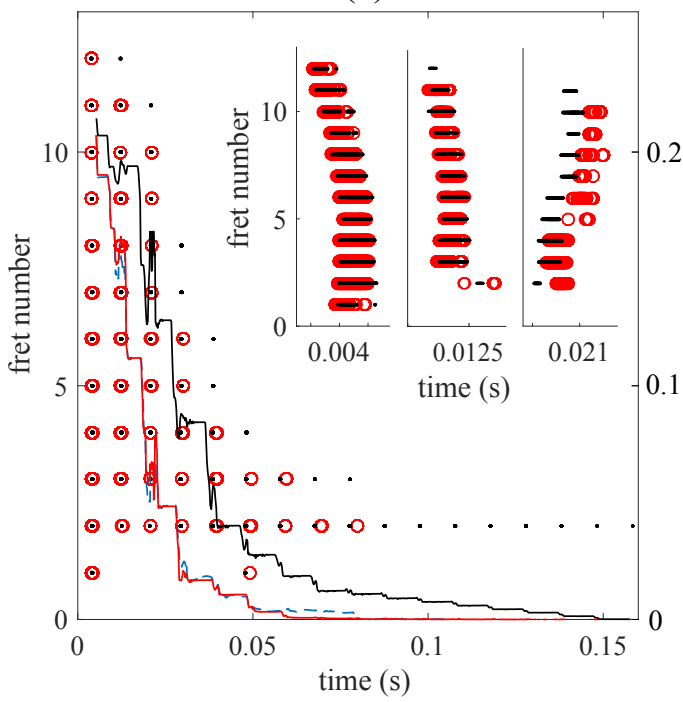

(b)

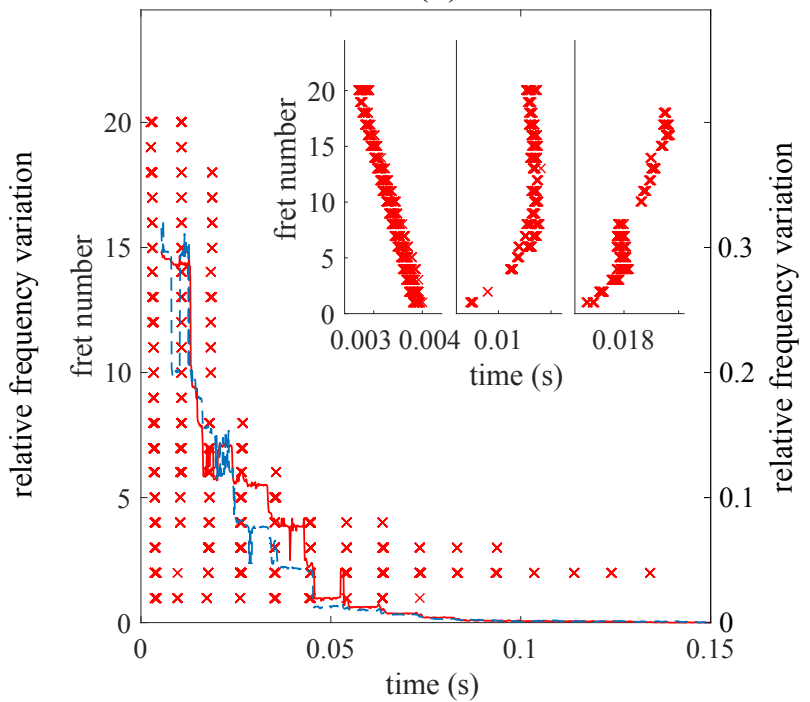

Figure 7: (a) Contact times for the numerical string with (red circles) and without (dark dots) dispersion. Relative fundamental frequency variation of the experimental signal (blue dashed line) and numerical results with (red line) and without dispersion (dark line). $u_{0, \max }=3.6 \mathrm{~mm}, F_{s}=8 \mathrm{MHz}$. Insert: zooms on the three first sets of contacts. (b) Contact times (red crosses) for the numerical string and relative fundamental frequency variation, experimental (dashed blue line) and numerical (red line). $u_{0, \max }=7.8 \mathrm{~mm}, F_{s}=16 \mathrm{MHz}$. Insert: zooms on the three first sets of contacts.
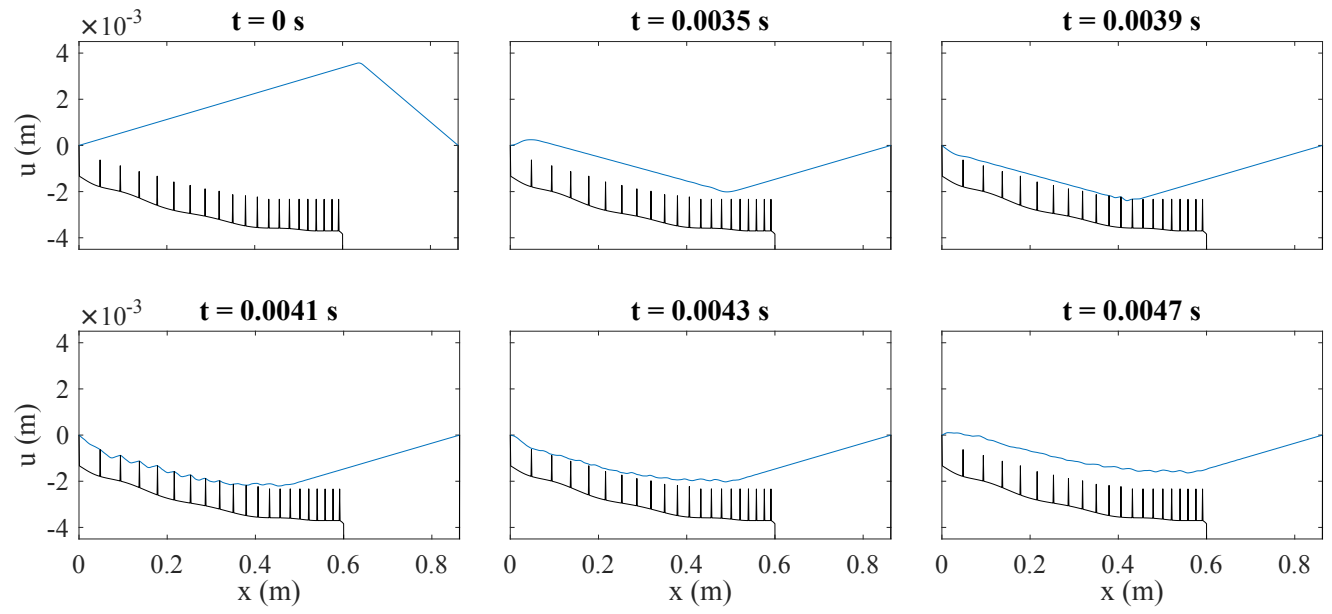

Figure 8: Snapshots of the numerical string displacement during the first period. $u_{0, \max }=3.6 \mathrm{~mm}, F_{s}=8 \mathrm{MHz}$. 
than in case B and lasts longer. This results in a different energy repartition compared to case B. The percussive part of the sound is very clearly visible at the beginning, and the overall sound is more nasal. This spectral content change is very well reproduced by the numerical simulation, and yields much higher characteristic frequencies during the whole signal, as can be seen in Fig. 6.

The contactogram in Fig. 7. b) confirms that more frets collide with the string for a higher excitation amplitude. During the first set of collisions, all the 20 frets are now hit. The staircase-like behaviour of the fundamental frequency is also retrieved, with now more differences between numerical and experimental signals, particularly around time $0.04 \mathrm{~s}$. Interestingly, the pitch glide is only driven by the contact times, and not by the geometrical nonlinearity which could have been involved as vibrations amplitudes are now moderate. This phenomenon being neglected in the model, our results in cases B and C clearly evidence that this fundamental frequency shift is not generated by nonlinearities associated with large-amplitude motions.

Finally, the three exposed cases show that the model employed, despite simplifying assumptions, includes the essential features for recovering the most important information present in the signal and more precisely in the attack transient. Our refined spectral and temporal investigations evidenced that a number of nonlinear characteristics are at hand during the vibration (spectral enrichment, pitch glide effects), which can be pertinently analysed in terms of contact times thanks to contactograms, and are satisfactorily reproduced numerically.

\subsubsection{Second polarisation}

This section is devoted to investigating the second polarisation by confronting experiments with simulations. For that purpose, an oblique initial condition with an angle of about 55 degrees between the excitation plane and $(x O y)$ is considered, so that energy is given to both transverse polarisations.

Fig. 9 shows temporal signals and spectrograms of both polarisations, of which maximal inital displacements are measured as $u_{0, \max }=3.1 \mathrm{~mm}$ along $(O z)$ and $v_{0, \max }=2.2 \mathrm{~mm}$ along $(O y)$. Friction force parameters (see Eq. (7)) are taken as $A=900 \mathrm{~N} \cdot \mathrm{m}^{-1}$ and $s=10^{-5} \mathrm{~m} . \mathrm{s}^{-1}$.

As in the previous section, the agreement is very satisfactory, both on temporal signals where details of the waveforms are finely reproduced, and on the spectrograms, displaying strong similarities. More specifically, for the horizontal displacement $v$, a low energy for the frequency about $2400 \mathrm{~Hz}$ and reinforced frequencies around 600,900 and $1400 \mathrm{~Hz}$ are observed in both experiment and simulation. Nevertheless, slight differences are also noticeable. They may be due to measurement uncertainties as already mentioned in the previous section. Moreover, in this case, it must be noticed that the shape of frets is assumed to be flat along the $(O y)$ direction in the model. A slight curvature is actually present, so that an enriched model with a non-constant obstacle along $(O y)$ or spatially varying coefficients in the friction law could be considered.

Finally, this comparison shows that with a very simple coupling model between the two polarisations through a friction mechanism, the model is able to retrieve the dynamics of the string with a number of collisions involved. It underlines that the most important physical features are taken into account in the model which can now be used for a parametric investigation.

\subsection{Application: parameters variations}

Sounds produced with pop and slap playing techniques strongly depend on materials of the string and fretboard, the fretboard shape and the player's gesture. The distance between strings and frets may be adjusted depending on musicians preferences, for instance a small distance may be more suitable to favour contacts if pop and/or slap are often intended. However this distance should be large enough to avoid undesirable grazing, therefore a compromise has to be found. With given materials and fretboard profile, a musician adapts its gesture to eventually favour contact, and to shape the sound by selecting a particular plucking point for instance.

In this section, in order to give a first insight into the influence of these parameters on the sound, the effects of contact parameters, dispersion, the plucking point and the fretboard profile are numerically studied and analysed.

\subsubsection{String dispersion}

The choice of the string, and more specifically its material properties and its diameter, leads to varying dispersion properties. Its influence on the attack transient is thus investigated here. 
(a)
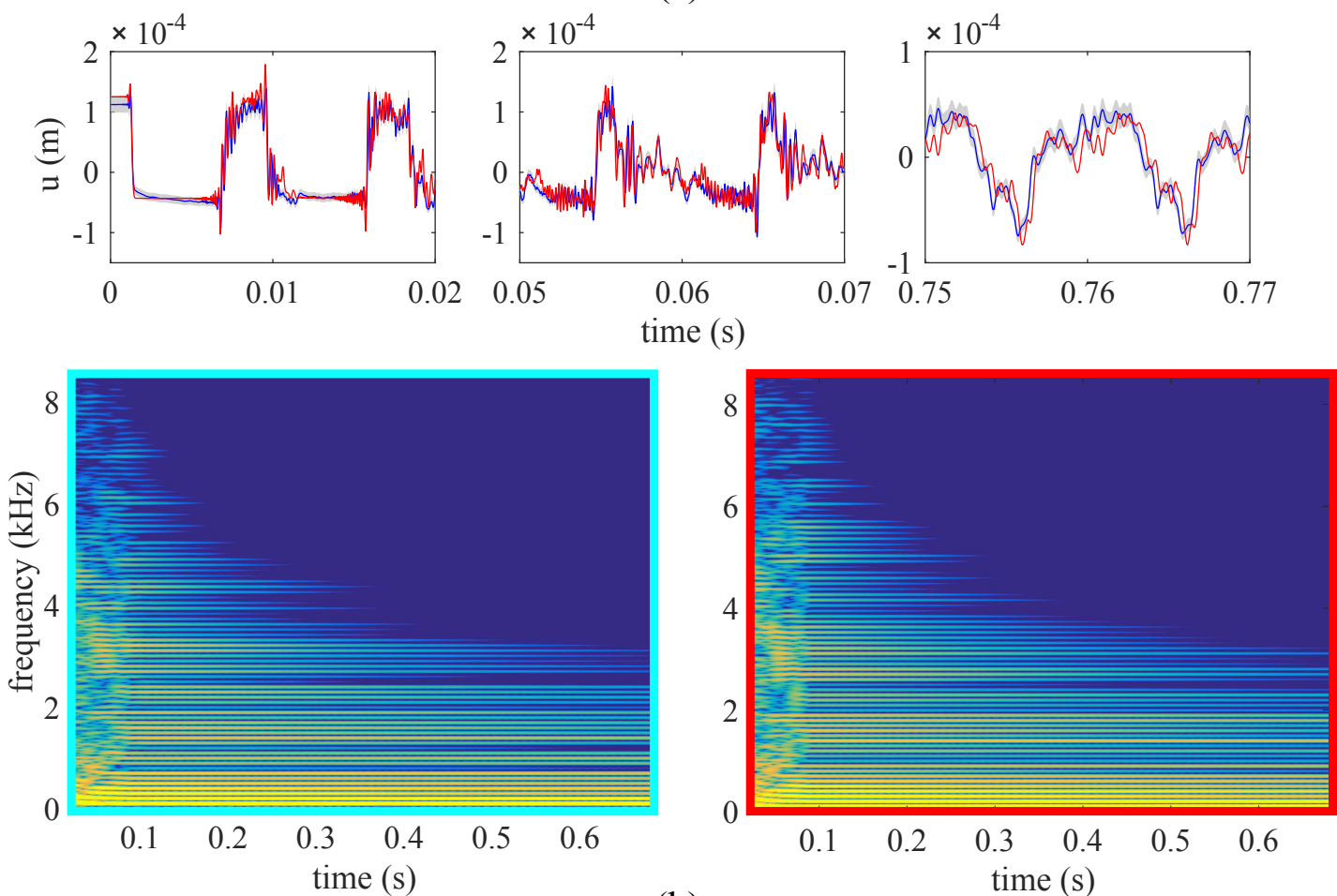

(b)
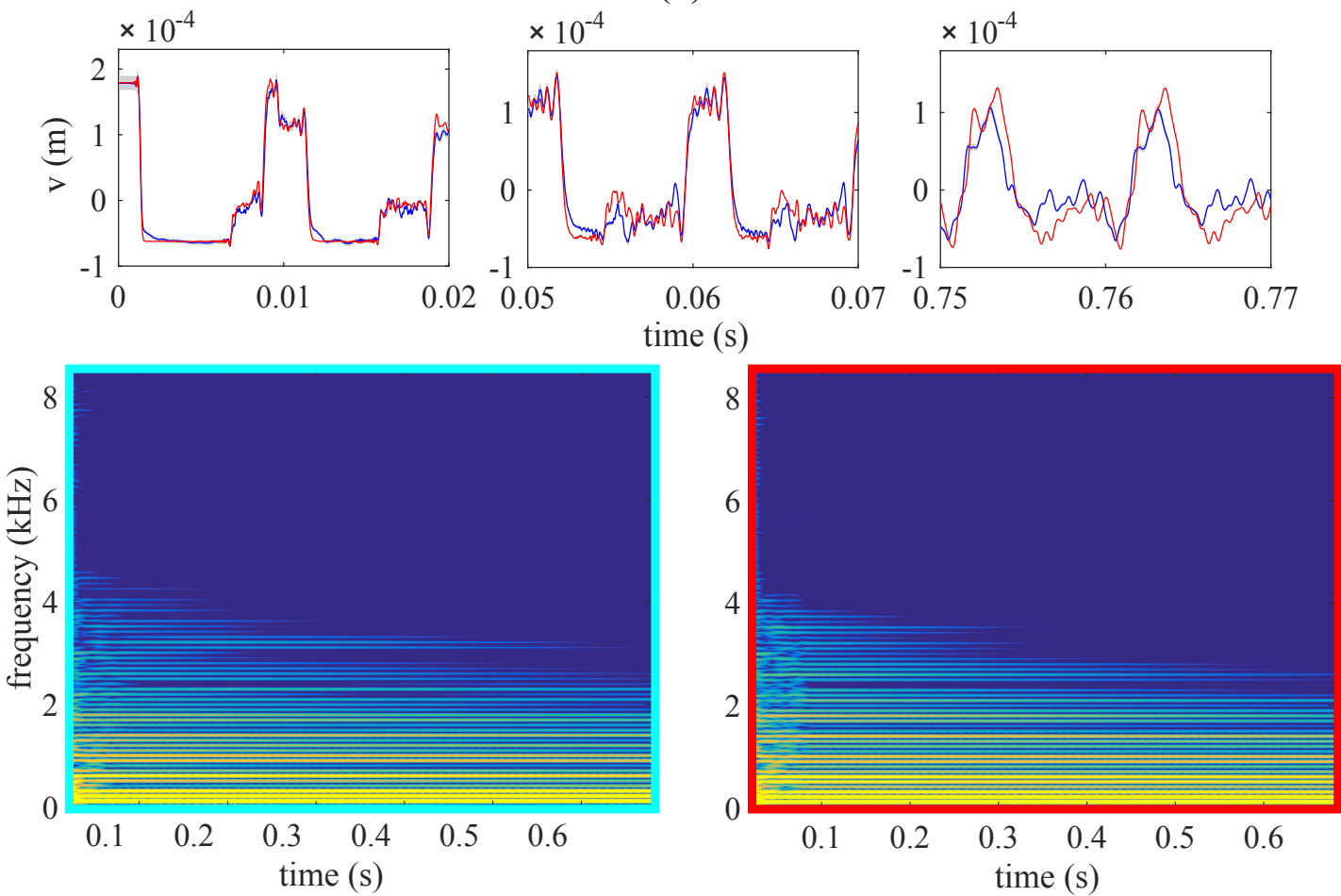

Figure 9: Displacement of the string with contact and spectrograms, two polarisations are excited. Comparison between measurement (blue line) and numerical simulation (red line). Expanded uncertainty at $95 \%$ (gray). Spectrogram of the experimental (left, blue frame) and numerical (right, red frame) signals. $u_{0, \max }=3.1 \mathrm{~mm}, v_{0, \max }=2.2 \mathrm{~mm}$, $F_{s}=8 \mathrm{MHz}$. (a) $u$, along $(O z)($ at $x=L-9 \mathrm{~mm})$, (b) $v$, along $(O y)$ (at $x=L-18 \mathrm{~mm}$ ). 


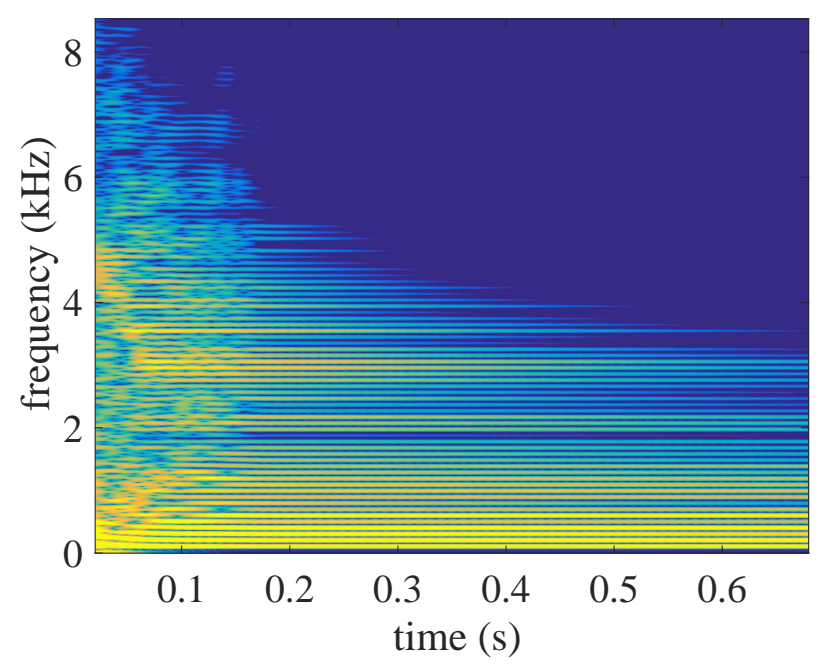

Figure 10: Spectrogram of the numerical signal without dispersion. $u_{0, \max }=3.6 \mathrm{~mm}, F_{s}=8 \mathrm{MHz}$.

Fig. 77.a) shows contact times as well as the fundamental frequency glide during the attack transient, with and without dispersion. From the third set of contacts (i.e. from about $0.02 \mathrm{~s}$ ), contacts arise earlier when dispersion is discarded. Moreover, contacts arise over a longer duration, and the number of hit frets decreases less rapidly than in the case with dispersion. Consequently the pitch glide also lasts longer, as evidenced by the fundamental frequency variation shown in Fig. 77.a). This is also observed in the spectrogram shown in Fig. 10, where the time interval in which contacts occur, characterised by complex spectral energy transfers, lasts about $0.15 \mathrm{~s}$.

This numerical experiment shows that dispersion, although important, is not as essential to describe the sound produced as in the case of tanpura for instance, as underlined e.g. in [14, 11. This may be due to the fact that in the tanpura string vibrations, contacts are present during the permanent regime as a key nonlinear feature characterising the timbre of the sound, whereas in the present case collisions only arise in the attack transient.

\subsubsection{A player's parameter: plucking point influence}

In this section, the effect of the plucking point is investigated. The same configuration as in case B of Section 4.1 is used, only the plucking point is modified. Three positions are tested: near the bridge (case a), in the middle of the string (case b), and near the nut (case c).

Fig. 11(a-c) shows the resulting spectrograms. The most significant consequence of the plucking position change is the length of the overall contact time interval, which is shorter in case $b$ and longer in case c. This is more clearly identified in Fig. 11(d) displaying the associated contactograms. When the string is plucked near the bridge and in the middle of the string, some frets collide at the beginning, before a global decrease of the number of hit frets arises. This behaviour is similar to cases $\mathrm{B}$ and $\mathrm{C}$ studied in Section 4.1. and leads to a percussive attack with more high frequencies as more contacts arise. When the string is plucked near the nut, a completely different behaviour appears. Some frets are hit at the beginning, their number then quickly reduces to one (the last fret) until no contact arises anymore between 0.25 and $0.35 \mathrm{~s}$. After this time, many contacts appear even after several tenths of seconds without contact, possibly from the beginning. For instance, the first contact between the string and the second fret occurs at time $0.85 \mathrm{~s}$. This specific behaviour leads to a grazing sound.

The studied cases evidence the influence of the plucking point on the produced sound: in order to increase the role of contacts, the musician may either pluck the string stronger (see Section 4 ) or at different positions. Even though it arises for an unusual plucking position here, the case resulting in a grazing sound (case c) is of particular interest, since it shows the possibility of a "growing" contact that leads to a generally undesirable sound. For standard playing conditions, avoiding this effect is of prime importance and is strongly related to the neck profile, which is a major issue in guitar making. 
(a)

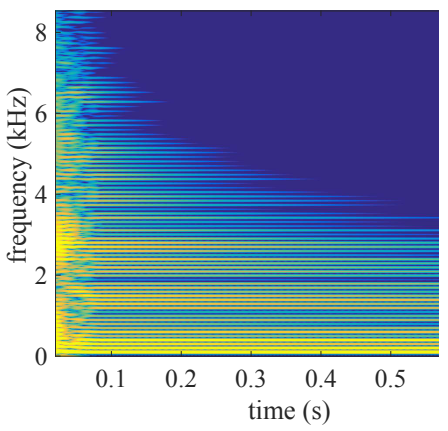

(b)
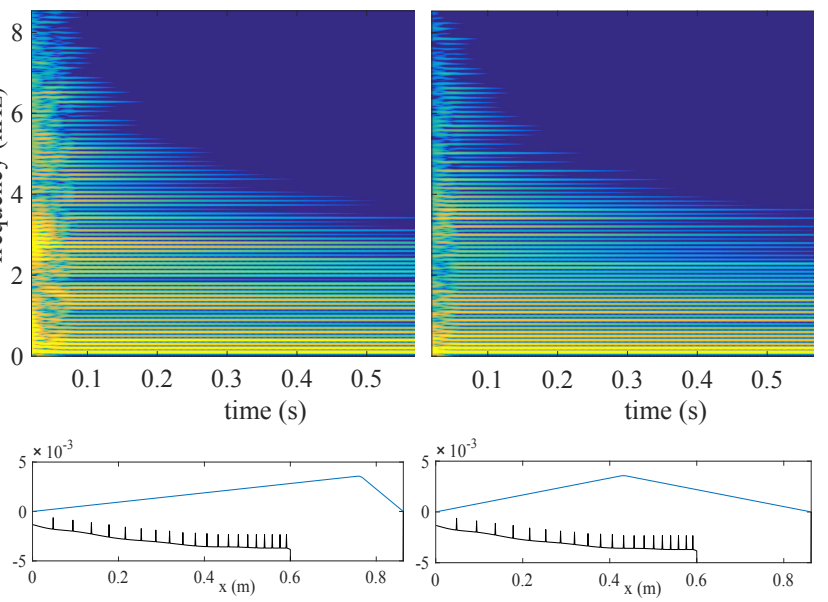

(c)
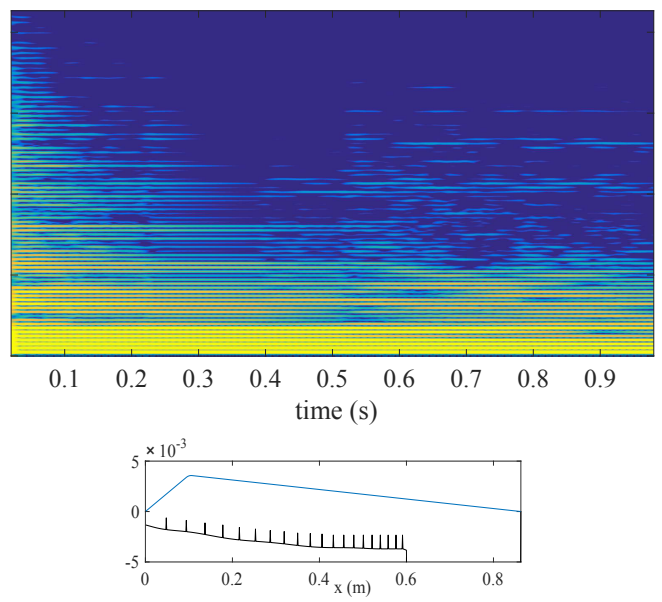

(d)
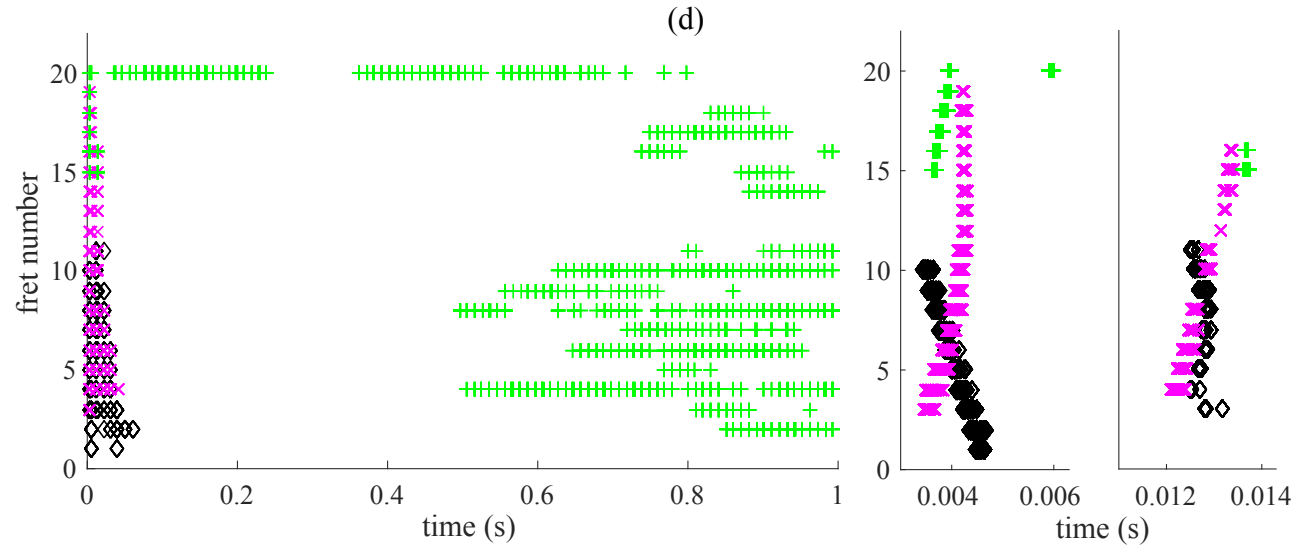

Figure 11: $u_{0, \max }=3.6 \mathrm{~mm}, F_{s}=4 \mathrm{MHz}$. (a-c) Top: Spectrograms of numerical signals, drawn in dB using a $70 \mathrm{~dB}$ dynamic. Bottom: Initial configurations (neck profile in dark and string position in blue). Plucking at: (a) $x=L-0.1$ $\mathrm{m}$ (b) $x=L / 2 \mathrm{~m}$ (c) $x=0.1 \mathrm{~m}$. (d) Contact times for the numerical string when plucking at $x=L-0.1 \mathrm{~m}(\mathrm{dark}$ diamonds), $x=L / 2 \mathrm{~m}$ (magenta crosses) and $x=0.1 \mathrm{~m}$ (green plus signs).

\subsubsection{A guitar maker's parameter: the neck adjustment}

In this section, a first insight is given into the neck shape influence in the case of a small initial displacement, so that no contact occurs with the measured fretboard, through two examples: the decrease of the neck curve and an abnormally raised fret.

Fig. 12 (a-c) shows spectrograms of string displacements for three fretboard curvatures. The first one is the measured fretboard, with a distance $d_{12}=2.3 \mathrm{~mm}$ between the string at rest and the 12th fret. Then, the fretboard curvature is reduced by rising frets inside the fretboard such that $d_{12}=2 \mathrm{~mm}$, the first one and the last one keeping the same height as previously. The third tested curvature corresponds to a straight fretboard, the straight line being based on the first and last measured frets heights. In this case, $d_{12}=1.8 \mathrm{~mm}$. The string is plucked with a $1.8 \mathrm{~mm}$ maximal initial displacement, at $60 \mathrm{~cm}$ from the nut, so that there is no contact with the measured fretboard as can be seen on the related spectrogram, in Fig. 12(a). When reducing the curvature, collisions arise as shown in Fig. 12(e). In the case of a straight fretboard, contacts arise earlier. Moreover, more frets are hit at the beginning, therefore the frequency glide starts from a higher frequency. On spectrograms, higher frequencies are involved. Furthermore, the highly perturbed zone at the beginning lasts longer and clearer reinforced spectral zones are visible.

Let us now consider the case of the measured fretboard on which the sixth fret would be raised by $0.5 \mathrm{~mm}$. As shown in Fig. 12(d-e), contacts with the sixth fret greatly alter the resulting sound, not only during the attack transient but also in the full length of the instrument sound.

The presented cases correspond to typical issues in guitar bass making, needing fine adjustments of the neck. They give a first insight into the way the model presented here might be considered for 
(a)
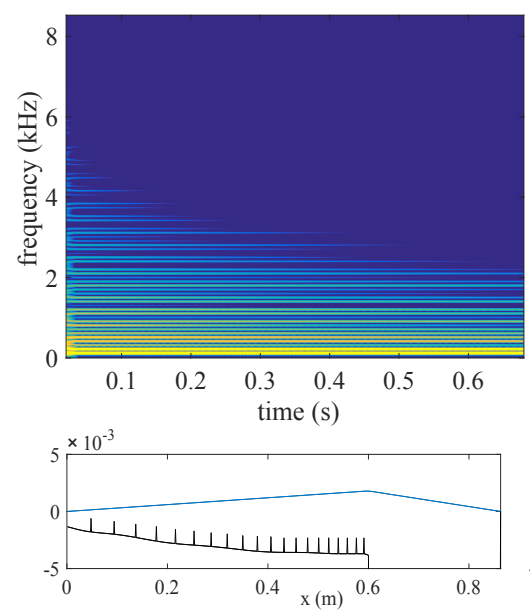

(d)
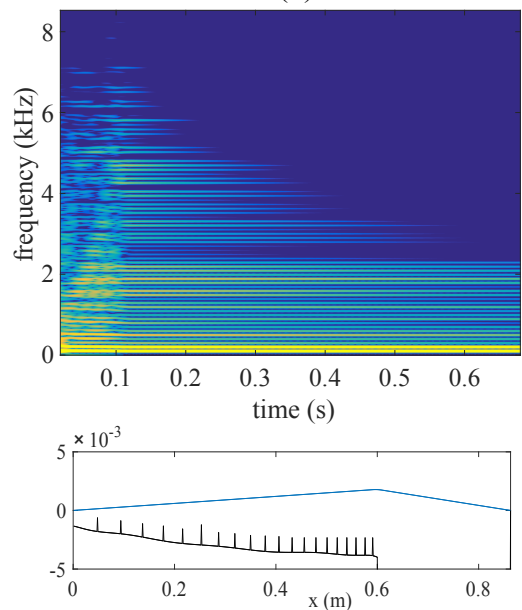

(b)
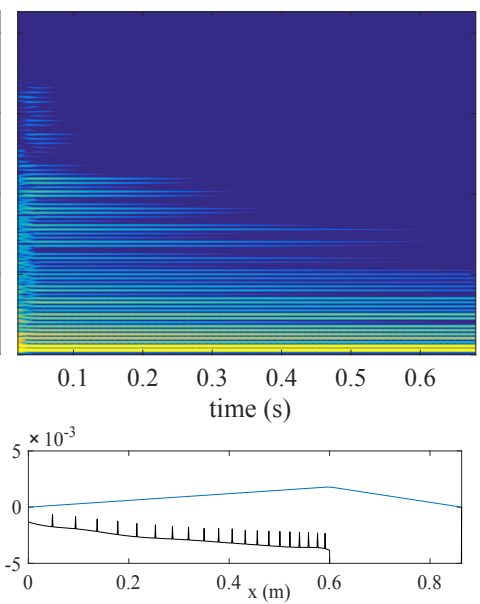

(e)

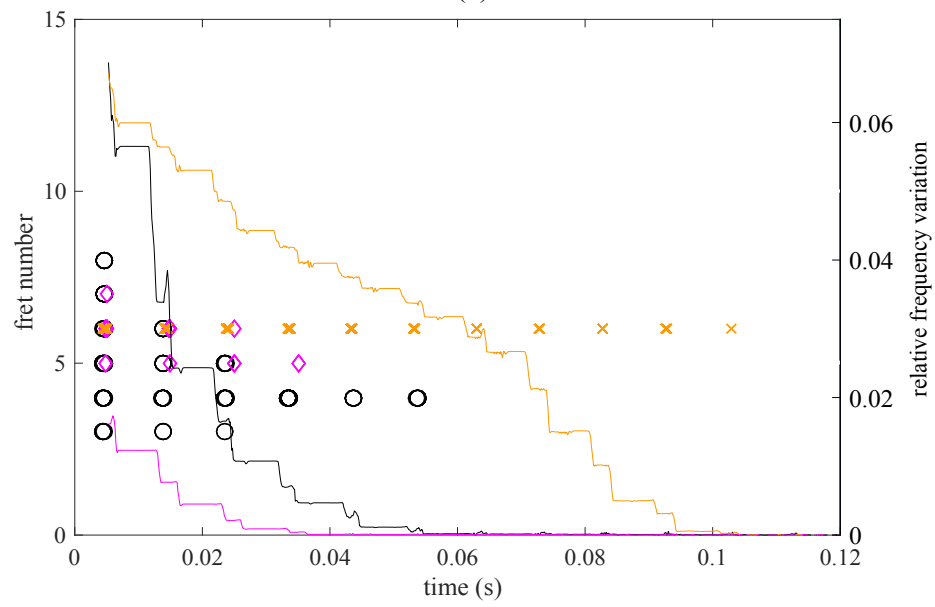

Figure 12: Top: Spectrograms of numerical signals, drawn in $\mathrm{dB}$ using a $70 \mathrm{~dB}$ dynamic. Bottom: Initial configuration (neck profile in dark and string position in blue). $u_{0, \max }=1.8 \mathrm{~mm}, F_{s}=2 \mathrm{MHz}$. (a) Measured profile (largest tested curvature) (b) Intermediate curvature (c) Straight profile (d) Sixth fret raised by $0.5 \mathrm{~mm}$. (e) Contact times for the numerical string with an intermediate curvature (magenta diamonds), a straight profile (dark circles) and the sixth fret raised (orange crosses). Relative fundamental frequency variation of the signal with an intermediate curvature (magenta line), a straight fretboard (dark line) and the sixth fret raised (orange line).

teaching purpose for instance.

\subsubsection{Contact stiffness}

In the simulations, the duration of the global contact period, corresponding to a complex transitory part, strongly depends on the obstacle position as well as the numerical stiffness of the contact. The smoother the contact is, the longer the time period with contacts lasts. For $K$ sufficiently small, for instance $K=10^{9}$, the previously mentioned frequency glide can be clearly heard during the contact period which then lasts several tenths of seconds, as shown in Fig. 13. The fundamental frequency estimation presents successive increases and decreases with an overall decrease during about $0.8 \mathrm{~s}$, with an evolution strongly related to the number of frets colliding with the string as shown in Fig. 13.(b). This leads to complex energy transfers, as can be seen in Fig. 13.a). Since contacts are smoother than for $K=10^{13}$, highest modes have less energy, as can be observed by comparing Fig. 13.a) and 5(a-b).

Contact parameters, which may be related to materials in contact [32, 33, participate to shaping the sound and may then be adjusted as materials properties. 
(a)

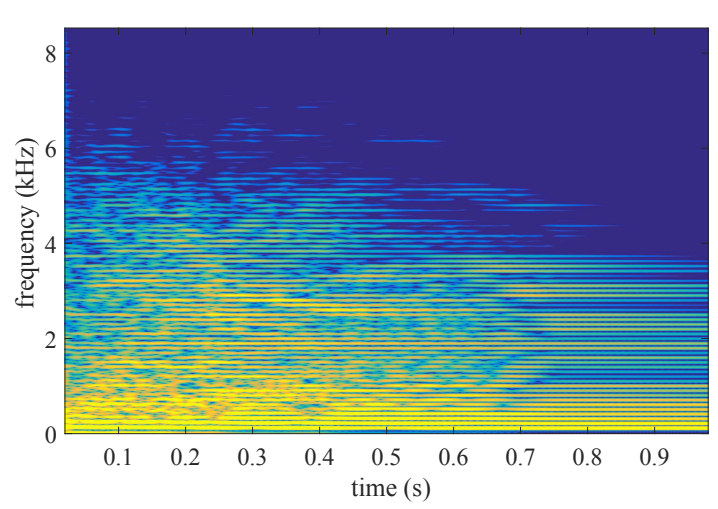

(b)

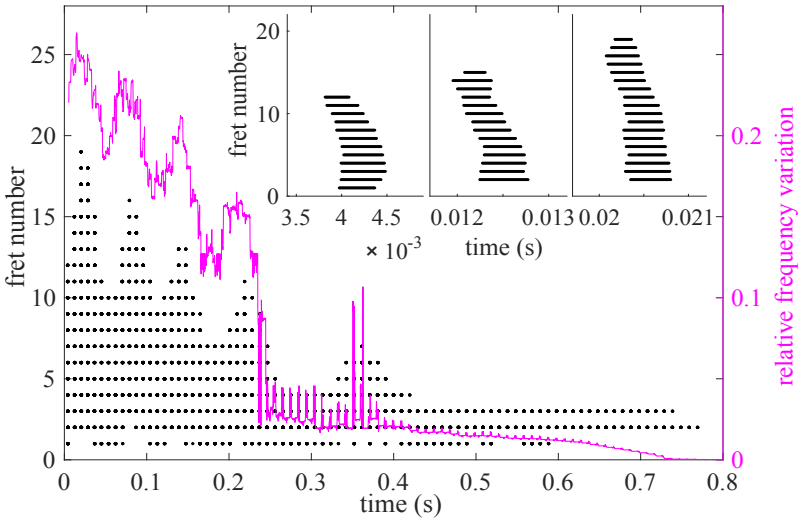

Figure 13: Numerical signal with $K=10^{9}, u_{0, \max }=3.6 \mathrm{~mm}, F_{s}=4 \mathrm{MHz}$. (a) Spectrogram of the numerical signal. (b) Contact times for the numerical string (dark dots) and relative fundamental frequency variation (magenta line).

\section{Conclusion}

In this study, a numerical modal-based model was applied to the string/fretboard contact in the case of a solid-body electric bass. Highly controlled experiments were conducted on this instrument, in order to compare data to numerical signals for both transverse polarisations. The model, despite its simplifying assumptions, faithfully and accurately reproduces the specific sound of the electric bass, possibly including contacts. Several specific patterns appear when contacts arise, among which complex energy transfers between modes and a frequency glide. Then the influence of numerical parameters as well as the role of the plucking point and neck tuning were numerically explored. In particular, the sensitivity of the sound to the height of frets was highlighted. These features may be adjusted to obtain either highly realistic or nonstandard sounds that could enrich a musical creation for instance.

This work might be extended to instruments with a different obstacle to the string vibration as fretless basses, and more realistic excitations may be included according to musicians' fingers gesture [34. The system may also be completed by considering all strings together, which involves sympathetic vibrations [26], as well as additional damping due to the presence of the musician and the effect of the microphone, through which the sound is transmitted [35].

\section{Acknowledgments}

The authors thank Laurent Quartier for contributing to the experimental set up realisation, JeanThéo Jiolat for preliminary experiments during his internship, and the guitar maker Yann-David Esmans for fruitful discussions. The authors also thank the reviewers of the manuscript for their careful reading which helped to improve the final version.

\section{References}

[1] T. Bacon, Electric bass guitar, http://www.oxfordmusiconline.com/subscriber/article/ grove/music/45729, grove Music Online. Oxford Music Online.Oxford University Press. Accessed: $2017 / 03 / 02$.

[2] H. Fleischer, Vibration of an electric bass guitar, Acta Acustica united with Acustica 91 (2005) $247-260$.

[3] A. Paté, J.-L. Le Carrou, B. Fabre, Predicting the decay time of solid body electric guitar tones, Journal of the Acoustical Society of America 135 (5) (2014) 3045-3055.

[4] A. Shipton, Slap-bass, http://www.oxfordmusiconline.com/subscriber/article/grove/ music/51117, grove Music Online. Oxford Music Online.Oxford University Press. Accessed: $2017 / 03 / 02$. 
[5] A. Krishnaswamy, J. O. Smith, Methods for simulating string collisions with rigid spatial objects, in: IEEE Workshop of Applications of Signal Processing to Audio and Acoustics, New Paltz, NY, 2003, pp. 233-236.

[6] G. Evangelista, F. Eckerholm, Player-instrument interaction models for digital waveguide synthesis of guitar: Touch and collisions, IEEE transactions on audio, speech, and language processing 18 (4) (2010) 822-832.

[7] D. Kartofelev, A. Stulov, H.-M. Lehtonen, V. Välimäki, Modeling a vibrating string terminated against a bridge with arbitrary geometry, in: Stockholm Music Acoustics Conference, 2013.

[8] V. Chatziioannou, M. van Walstijn, Energy conserving schemes for the simulation of musical instrument contact dynamics, Journal of Sound and Vibration 339 (2015) 262-279.

[9] S. Bilbao, A. Torin, V. Chatziioannou, Numerical modeling of collisions in musical instruments, Acta Acustica united with Acustica 101 (2015) 155-173.

[10] M. van Walstijn, J. Bridges, Simulation of distributed contact in string instruments: a modal expansion approach, European Signal Processing Conference (EUSIPCO) (2016) 1023-1027.

[11] C. Issanchou, S. Bilbao, J.-L. Le Carrou, C. Touzé, O. Doaré, A modal-based approach to the nonlinear vibration of strings against a unilateral obstacle: simulations and experiments in the pointwise case, Journal of Sound and Vibration 393 (2017) 229-251.

[12] C. Valette, C. Cuesta, Mécanique de la corde vibrante, Hermès, Paris, 1993.

[13] C. P. Vyasarayani, S. Birkett, J. McPhee, Modeling the dynamics of a vibrating string with a finite distributed unilateral constraint: Application to the sitar, Journal of the Acoustical Society of America 125 (6) (2009) 3673-3682.

[14] S. Siddiq, A physical model of the nonlinear sitar string, Archives of acoustics 37 (1) (2012) 73-79.

[15] E. Rank, G. Kubin, A waveguide model for slapbass synthesis, in: IEEE International Conference on Acoustics, Speech, and Signal Processing, Munich, Vol. 1, 1997, pp. 443-446.

[16] P. Kramer, J. Abeßer, C. Dittmar, G. Schuller, A digital waveguide model of the electric bass guitar including different playing techniques, IEEE International Conference on Acoustics, Speech and Signal Processing (2012) 353-356.

[17] C. Cuesta, C. Valette, Théorie de la corde pincée en approximation linéaire, Acustica 71 (1990) $28-40$.

[18] V. Acary, B. Brogliato, Numerical methods for nonsmooth dynamical systems. Applications in mechanics and electronics, Vol. 35 of Lecture Notes in Applied and Computational Mechanics, Springer Verlag, 2008.

[19] F. Chouly, An adaptation of Nitsche's method to the Tresca friction problem, Journal of Mathematical Analysis and Applications 411 (2014) 329-339.

[20] M. Hirschkorn, J. McPhee, S. Birkett, Dynamic modeling and experimental testing of a piano action mechanism, Journal of computational and nonlinear dynamics 1 (2005) 47-55.

[21] J. Bridges, M. Van Walstijn, Investigation of tanpura string vibrations using a two-dimensional time-domain model incorporating coupling and bridge friction, in: Vienna Talk on Music Acoustics, Vienna, 2015.

[22] A. Thorin, Non-smooth model of the grand piano action, Ph.D. thesis, Ecole polytechnique (2013).

[23] C. Issanchou, S. Bilbao, J.-L. Le Carrou, C. Touzé, O. Doaré, A modal aproach to the numerical simulation of a string vibrating against an obstacle: applications to sound synthesis, in: International Conference on Digital Audio Effects, DAFx-16, Brno, 2016. 
[24] T. Catterou, V. Blanc, G. Ricciardi, S. Bourgeois, B. Cochelin, Méthode de projection modale appliquée à un problème de dynamique multi-contact, in: Colloque national en calcul des structures, CSMA 2017, Giens, 2017.

[25] J.-L. Le Carrou, D. Chadefaux, L. Seydoux, B. Fabre, A low-cost high-precision measurement method of string motion, Journal of Sound and Vibration 333 (2014) 3881-3888.

[26] J.-L. Le Carrou, F. Gautier, N. Dauchez, J. Gilbert, Modelling of sympathetic string vibrations, Acta Acustica united with Acustica 91 (2) (2005) 277-288.

[27] R. Roy, A. Paulraj, T. Kailath, Esprit - a subspace rotation approach to estimation of parameters of cisoids in noise, IEEE transactions on acoustics, speech, and signal processing 34 (5) (1986) $1340-1342$.

[28] J.-L. Le Carrou, F. Gautier, R. Badeau, Sympathetic string modes in the concert harp, Acta Acustica united with Acustica 95 (4) (2009) 744-752.

[29] M. Ducceschi, O. Cadot, C. Touzé, S. Bilbao, Dynamics of the wave turbulence spectrum in vibrating plates: A numerical investigation using a conservative finite difference scheme, Physica D 280-281 (2014) 73-85.

[30] D. Kartofelev, A. Stulov, V. Välimäki, Pitch glide effect induced by a nonlinear string-barrier interaction, in: AIP Conference, 2015.

[31] A. de Cheveigné, H. Kawahara, Yin, a fundamental frequency estimator for speech and music, The Journal of the Acoustical Society of America 111 (4) (2002) 1917-1930.

[32] M. Machado, P. Moreira, P. Flores, H. M. Lankarani, Compliant contact force models in multibody dynamics: Evolution of the Hertz contact theory, Mechanism and Machine Theory 53 (2012) 99121.

[33] A. Banerjee, A. Chanda, R. Das, Historical origin and recent development on normal directional impact models for rigid body contact simulation: A critical review, Archives of Computational Methods in Engineering (2016) 1-26.

[34] D. Chadefaux, J.-L. Le Carrou, B. Fabre, A model of harp plucking, Journal of the Acoustical Society of America 133 (4) (2013) 2444-2455.

[35] A. Novak, L. Guadagnin, B. Lihoreau, P. Lotton, E. Brasseur, L. Simon, Non-linear identification of an electric guitar pickup, in: International Conference on Digital Audio Effects, DAFx-16, Brno, 2016. 